\title{
Celiac Disease and Focal Segmental Glomerulosclerosis: Is It a Fortuity Finding?
}

\author{
Hassan Harbi ${ }^{\mathrm{a}, \mathrm{d}}$, Mohamed Said Abdelsalam ${ }^{\mathrm{b}}$, Ahmed Abdulshakour ${ }^{\mathrm{a}}$, \\ Osman Alfurayh ${ }^{\mathrm{c}}$
}

\begin{abstract}
Celiac disease (CD) is an autoimmune disorder of the small intestine that occurs in genetically predisposed people of all ages and associated with an increased risk of developing other autoimmune conditions including type 1 diabetes mellitus, thyroid disease, Sjogren's syndrome and IgA nephropathy; nonetheless, focal segmental glomerulosclerosis (FSGS) collapsing variant was not reported before as one of glomerulonephritis associated with celiac disease. We report a case of nephrotic syndrome due to focal segmental glomerulosclerosis collapsing variant in a patient with $\mathrm{CD}$. An 18-year-old male patient presented with progressive generalized body swelling for 1 month. He was diagnosed with CD when proven by small bowel biopsy and FSGS collapsing type by renal biopsy. Patient was treated with gluten-free diet and immunosuppressive therapy. The association between $\mathrm{CD}$ and nephrotic syndrome is extremely rare. To the best of our knowledge, an association of CD with FSGS has been reported only in one case. Our extensive literature review revealed no reported cases stating association of $\mathrm{CD}$ and appearances of collapsing variant of FSGS. The query arises whether there is a rare association between $\mathrm{CD}$ and FSGS, or this is a fortuity finding?
\end{abstract}

Keywords: Celiac disease; Focal segmental glomerulosclerosis; Focal segmental glomerulosclerosis collapsing type; Association

\section{Introduction}

Celiac disease $(\mathrm{CD})$ is one of the most common dietary-medi-

\footnotetext{
Manuscript accepted for publication October 23, 2015

${ }^{a}$ Internal Medicine Department, King Faisal Specialist Hospital \& Research Center, Riyadh, KSA

bNephrology Unit, Internal Medicine Department, Faculty of Medicine, Alexandria University, Alexandria, Egypt

${ }^{c}$ Nephrology Department, King Faisal Specialist Hospital \& Research Center, Riyadh, KSA

${ }^{\mathrm{d} C}$ Corresponding Author: Hassan Mohammed Al-Harbi, Internal Medicine Department, King Faisal Specialist Hospital \& Research Center, Riyadh, KSA. Email: hharbi64@kfshrc.edu.sa
}

doi: http://dx.doi.org/10.14740/wjnu242w ated inflammatory enteropathies that occur in genetically predisposed individuals. Genetic factors (HLA-DQ2 and/or DQ8 antigens) are involved in its pathogenesis, as well as environmental factors, and there are changes in humoral and cellular immunity responses to antigens contained in cereals (wheat, barley, rye and oats) which contain a protein called gluten. The peptides derived from gliadin (the alcohol-soluble fraction of gluten) possess many toxic components, which contain various antigens implicated in its pathogenesis [1]. CD is associated with a variety of autoimmune diseases such as type 1 diabetes mellitus, autoimmune thyroid disorders, Sjogren's syndrome and IgA nephropathy [2]. Focal segmental glomerulosclerosis (FSGS) is a cause of nephrotic syndrome in children and adolescents, as well as an important cause of renal failure in adults [3]. It is also known as "focal glomerular sclerosis" or "focal nodular glomerulosclerosis". It accounts for about one-sixth of the cases of nephrotic syndrome [4]. FSGS arises due to either idiopathic (primary) or secondary causes. Focal proliferative glomerulonephritis (such as IgA nephropathy, lupus nephritis, pauci-immune focal necrotizing and crescentic glomerulonephritis) is a cause of secondary FSGS [5]. IgA nephropathy, immune complex-associated or membranoproliferative glomerulonephritis are the most common forms of glomerulonephritis in patients with CD [6]. There are variants of FSGS, and collapsing glomerulopathy is one of the morphologic variants of focal segmental glomerulosclerosis (CG-FSGS) characterized by segmental and global collapse of the glomerular capillaries, marked hypertrophy and hyperplasia of podocytes, and severe tubulointerstitial disease [7]. The cause of this disorder is unknown, but is seen in association with HIV infection and increasingly recognized in non-HIV patients. Some of nonHIV patients are associated with systemic lupus erythematosus (SLE)-like disease, lymphoproliferative disorders, and viral infections [8]. However, CD is not known among one of these associations.

\section{Case Report}

The patient is an 18-year-old male patient from south region of Saudi Arabia. He is known to have CD on gluten-free diet only based on clinical presentation and small intestinal biopsy 2 year ago. He is a short stature young man, and was not on any medication before with no family history of kidney disease/ 


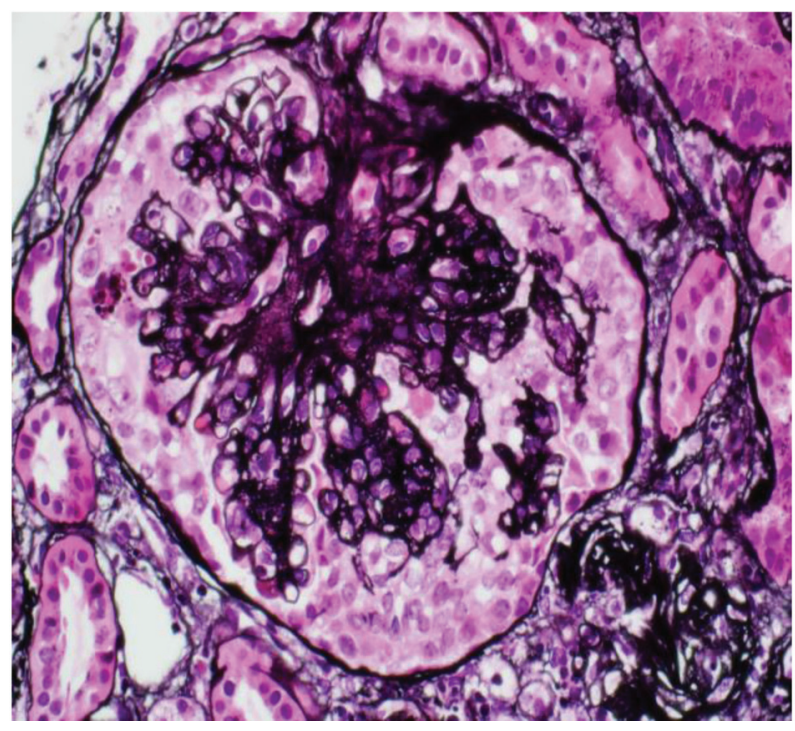

Figure 1. Renal biopsy.

autoimmune disease. He came to our hospital with history of generalized body swelling with blood pressure 118/72 $\mathrm{mm} \mathrm{Hg}$. Lab showed blood urea nitrogen (BUN) of $9.9 \mathrm{mg} / \mathrm{dL}$, creatine of $124 \mathrm{mmol} / \mathrm{L}$ and hemoglobin of $14.6 \mathrm{~g} / \mathrm{L}$. Additional testing revealed a low serum albumin of $10.1 \mathrm{~g} / \mathrm{L}$ and proteinuria of $6 \mathrm{~g}$ /day; antinuclear antibody (ANA), complement 3 (C3) and complement 4 (C4) were normal. Hepatitis B, C serology and HIV screen were also negative. Renal biopsy was done and showed renal cortex with 12 glomeruli present for evaluation. None revealed global sclerosis and three showed lesion of segmental sclerosis with collapsing features. The remaining glomeruli showed normal architecture with normal thickness of capillary walls and patent capillary lumina. There is mild multifocal interstitial fibrosis and tubular atrophy (15-20\% of cortical tissue), associated with minimal focal interstitial lymphocyte infiltration. Vascular compartment is within normal limits and on electron microscopy (EM13-679), the semithin section revealed one glomerulus (Fig. 1). Ultrastructure examination demonstrated peripheral capillary walls with normal thickness, contours and texture, mesangial areas are unremarkable, and no electron dense deposits are seen. The podocytes showed diffuse foot process effacement. Patient was started on lisinopril $5 \mathrm{mg}$ to control the proteinuria and furosemide $40 \mathrm{mg}$ BID for the edema, along with atorvastatin $20 \mathrm{mg}$ oral daily. Prednisone $60 \mathrm{mg}$ /day was used, after 2 months followup, symptoms and laboratory results started to improve.

\section{Discussion}

Two decades ago, CD was considered a relatively uncommon disorder, with prevalence rates of 1 in 1,000 or lower $[8,9]$. But several recent population-based studies have shown a much increasing prevalence, and it is now estimated that CD may affect 1 in 100 to 200 individuals [1, 10]. The seroprevalence rate of $2.2 \%$ ( 1 in 45 ) found in Saudi Arabia citizen might be one of the highest seroprevalence rates of $\mathrm{CD}$ in the world, attributed to the high levels of consanguinity and the heavy gluten intake in Saudi Arabia [11].

Gimenez et al reported five cases of nephrotic syndrome in children associated with CD [12]. A few cases of CD associated with membranous nephropathy in adults have also been reported [13-15]. To the best of our knowledge, an association of CD with FSGS has been reported in only one case of an 83-year-old lady who had FSGS-associated polycythemia and CD [16]. Instated association between CD and nephrotic syndrome is extremely rare. Our extensive wide literature review shows no reported cases stating association of $\mathrm{CD}$ and appearances of collapsing variant of FSGS. The query arises as to whether CD and FSGS is an association or rare cause. In view of the fact that both are immune-mediated diseases, an association is possible but needs further extensive studies and therefore it is of significance to highlight this rare probable relation, as undiagnosed CD patients may be exposed to the risk of long-term complications [17]. Thus, it is important for primary care physicians, internists, gastroenterologists and nephrologists to consider probable relation when working up a patient with $\mathrm{CD}$ and renal failure.

\section{Conclusion}

The association between $\mathrm{CD}$ and nephrotic syndrome is extremely rare, and no reported cases declare association involving $\mathrm{CD}$ and collapsing variant of FSGS. The query arises as to whether CD and FSGS is an association or a rare cause.

\section{Competing Interests}

We hereby attest that all authors meet criteria for authorship and do not have any financial or non-financial conflicts of interest. We also attest that we do not have any funding from or shares in organizations that stand to gain or lose from the publication of this case.

\section{Abbreviations}

CG-FSGS: collapsing glomerulopathy - focal segmental glomerulosclerosis; BUN: blood urea nitrogen; ANA: antinuclear antibody; C3: complement 3; C4: complement 4; EM: electron microscopy; CD: celiac disease

\section{References}

1. Green PH, Cellier C. Celiac disease. N Engl J Med. 2007;357(17):1731-1743.

2. Lima VM, Gandolfi L, Pires JA, Pratesi R. Prevalence of celiac disease in dyspeptic patients. Arq Gastroenterol. 2005;42(3):153-156.

3. Kumar V, Fausto N, Abbas A, ed. Robbins \&Cotran Pathologic Basis of Disease(7th ed.). Saunders. 2003; pp. 982-983. ISBN 978-0-7216-0187-8. 
4. Renal Pathology. Retrieved 2008-11-25.

5. D'Agati VD, Fogo AB, Bruijn JA, Jennette JC. Pathologic classification of focal segmental glomerulosclerosis: a working proposal. Am J Kidney Dis. 2004;43(2):368382 .

6. Johnston SD, Watson RG, McMillan SA, Sloan J, Love AH. Prevalence of coeliac disease in Northern Ireland. Lancet. 1997;350(9088):1370.

7. Schwimmer JA, Markowitz GS, Valeri A, Appel GB. Collapsing glomerulopathy. Semin Nephrol. 2003;23(2):209218.

8. Laurinavicius A, Hurwitz S, Rennke HG. Collapsing glomerulopathy in HIV and non-HIV patients: a clinicopathological and follow-up study. Kidney Int. 1999;56(6):2203-2213.

9. Feighery C. Fortnightly review: coeliac disease. BMJ. 1999;319(7204):236-239.

10. Maki M, Mustalahti K, Kokkonen J, Kulmala P, Haapalahti M, Karttunen T, Ilonen J, et al. Prevalence of Celiac disease among children in Finland. N Engl J Med. 2003;348(25):2517-2524.

11. Aljebreen AM, Almadi MA, Alhammad A, Al Faleh FZ. Seroprevalence of celiac disease among healthy adolescents in Saudi Arabia. World J Gastroenterol. 2013;19(15):2374-2378.

12. Gimenez Llort A, Vila Cots J, Camacho Diaz JA, Vila Santandreu A, Concheiro Guisan A, Garcia Garcia L. Nephrotic syndrome associated with Celiac disease. A report of five cases. Nephron. 2002;92(4):950.

13. Casella G, Perego D, Baldini V, Monti C, Crippa S, Buda CA. A rare association between ulcerative colitis (UC), celiac disease (CD), membranous glomerulonephritis, leg venous thrombosis, and heterozygosity for factor V Leiden. J Gastroenterol. 2002;37(9):761-762.

14. Halma C, Ubels F. Celiac sprue-associated membranous nephropathy. Clin Nephrol. 2007;68(3):197.

15. Prasad D, Khara HS, Gupta M, Sterman P. Celiac disease associated membranous nephropathy - a rare cause or coincidence? A case report. Cases J. 2009;2:7018.

16. Martin JS, Suarez LG, Martin FG. [Focal and segmental glomerulosclerosis associated with polycythemia vera]. Nefrologia. 2010;30(1):138-140.

17. Ventura A, Neri E, Ughi C, Leopaldi A, Citta A, Not T. Gluten-dependent diabetes-related and thyroid-related autoantibodies in patients with celiac disease. J Pediatr. 2000;137(2):263-265. 